\title{
Self-Blotting Nanowire Grids for Cryo-EM Sample Preparation
}

Hui Wei, Venkat Dandey, Zhening Zhang, Ashleigh Raczkowski, Bridget Carragher, Clinton S. Potter National Resource for Automated Molecular Microscopy, Simons Electron Microscopy Center, New York Structural Biology Center, New York, USA

Abstract:

Almost every aspect of cryo electron microscopy (CryoEM) has been automated over the last few decades. One of the challenges that remains to be addressed is the robust and reliable preparation of vitrified specimens of suitable ice thickness. The development of a new self-blotting nanowire (Zhang et al., 2013) grid in conjunction with a piezo electric dispensing robot called Spotiton (Jain et al., 2012) enables spreading a sample to a thin film without the use of externally applied filter paper. This new approach has the advantage of using small amounts of protein material, resulting in large areas of ice of a well- defined thickness containing evenly distributed particles (Razinkov et al., 2016).

In the previously published work, we used a lacey carbon substrate. We have now modified our protocol to make lacey gold substrates (figure 1), which provide several advantages. Gold substrates are more stable when exposed to the electron beam (Russo and Passmore, 2016) which reduces beam induced motion, particularly at high tilt angles. This enables the nanowire grids to be used for tomography and collecting titled images to overcome preferred particle orientation (figure 2). Many particles also do not show a preference to adhere to a gold substrate, which is common for carbon substrates, enabling lower sample concentration to be effective in getting particles into the holes. Finally, the gold grids result in exceptionally even and thin vitreous ice (figure 3).

We will describe the protocols used to make the nanowire grids, and to vitrify samples using them. We will compare and contrast the results obtained from these grids with those from nanowire lacey carbon grid and grids made using traditional methods.

References:

1. Zhang, F., Zhang, W.B., Shi, Z., Wang, D., Jin, J., Jiang, L., 2013. Nanowire-haired inorganic membranes with superhydrophilicity and underwater ultralow adhesive superoleophobicity for highefficiency oil/water separation. Adv. Mater. 25, 4192-4198.

2. Jain, T., Sheehan, P., Crum, J., Carragher, B., Potter, C.S., 2012. Spotiton: a prototype for an integrated inkjet dispense and vitrification system for cryo-TEM. J. Struct. Biol. 179, 68-75.

3. Razinkov I, Dandey VP, Wei H, Zhang Z, Melnekoff D, Rice WJ, Wigge C, Potter CS, Carragher B. 2016. A new method for vitrifying samples for cryoEM. J Struct Biol. 195(2):190-8.

4. Russo, C.J., Passmore, L.A., 2016. Ultrastable gold substrates: properties of a support for highresolution electron cryomicroscopy of biological specimens. J. Struct. Biol. 193, 33-44. 


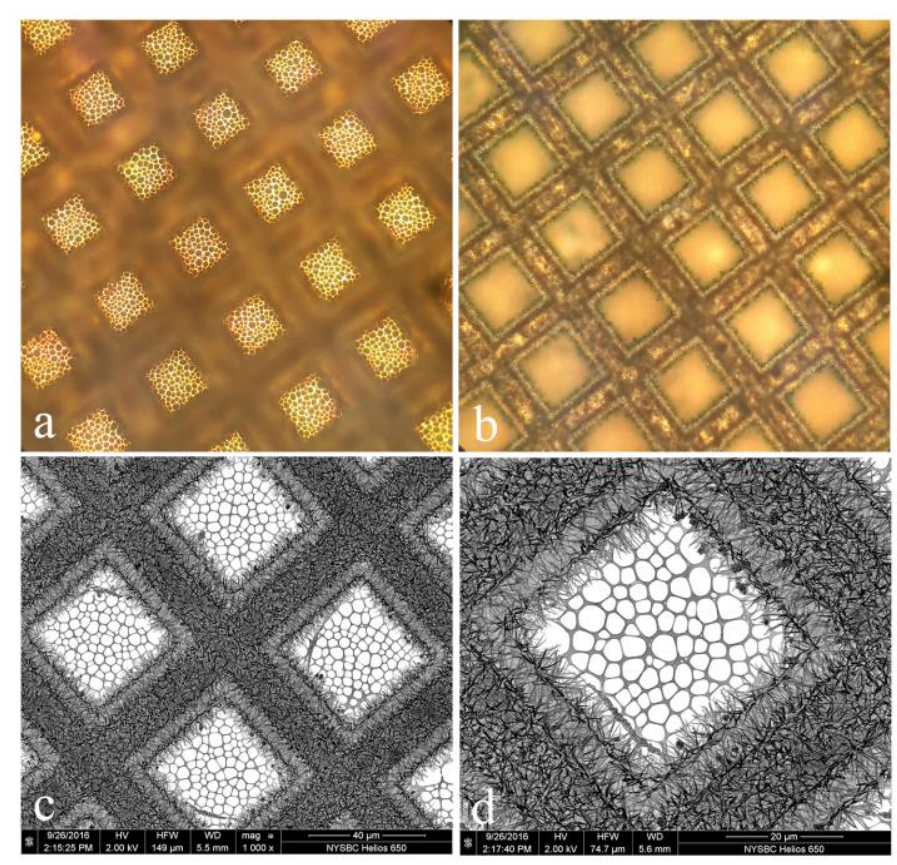

Figure 1: Gold lacey substrates on nanowire grid. Light microscopy image focused on gold substrate (a) and nanowire (b); (c, d) SEM images at two different magnifications.

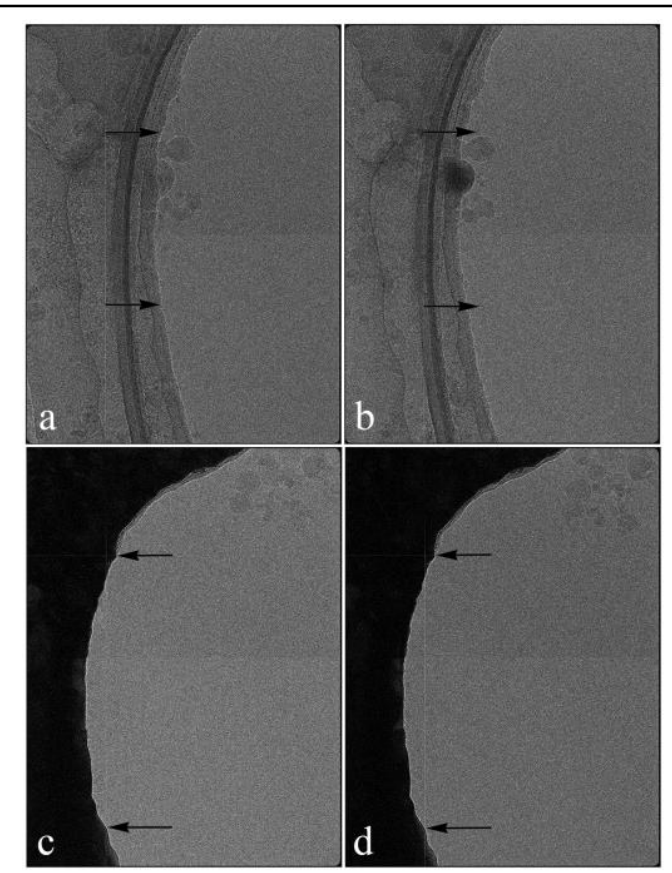

Figure 2: Stability comparison between gold and carbon substrates. Left are the first and right are the last frames of a 50 frame movie; the arrows are fixed fiducials.

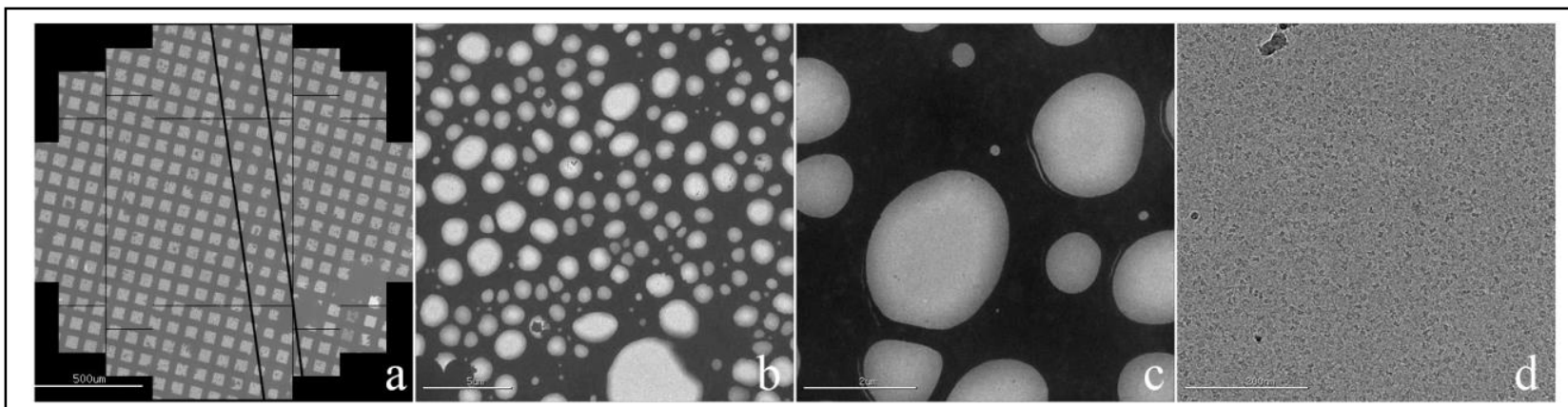

Figure 3: Sequentially increasing magnification images of a vitrified sample prepared using a gold lacey supporting film on a nanowire grid. (a) Grid view; the black rectangle indicates that a strip of vitreous ice deposited by Spotiton. (b, c, and d) are the sequential images showing that the ice is of consistently good quality and particles are well distributed. 